\title{
Spontaneous Aharonov-Casher effect of neutral hard-core bosons in one-dimensional mesoscopic rings
}

\author{
Z. D. Wang and Jian-Xin Zhu \\ Department of Physics, University of Hong Kong, Pokfulam Road, Hong Kong
}

(Received 17 January 1995)

\begin{abstract}
Using a tight-binding model Hamiltonian and applying the Jordan-Wigner transformation, we have investigated the Aharonov-Casher (AC) effect for many neutral hard-core bosons in onedimensional (1D) mesoscopic rings with the self-induced $\mathrm{AC}$ phase included. The total energy and the persistent fluxon current are analytically derived for $1 \mathrm{D}$ perfect lattices. More importantly, it is suggested that, in the absence of the external AC flux, the self-sustained AC flux state could be the ground state of a system with weak disorder. The possibility of experimental observations is briefly discussed.
\end{abstract}

With advances in nanotechnology, the persistent equilibrium current occurring in isolated mesoscopic normalmetal rings penetrated by an Aharonov-Bohm ${ }^{1}$ (AB) flux $\Phi_{\mathrm{AB}} \equiv \oint_{C} \boldsymbol{A} \cdot d \boldsymbol{l}$, where $\boldsymbol{A}$ is the vector potential, is one of the most interesting phenomena in mesoscopic systems. ${ }^{2,3}$ Considering that the persistent current itself also produces magnetic flux in addition to the externally applied flux, the possibility of a self-sustained (or spontaneous) persistent current for many-fermion systems was recently addressed. ${ }^{4}$ Interestingly, there also exists a topological interference effect - the AharonovCasher (AC) effect, ${ }^{5}$ which is an electromagnetic dual of the AB effect: a particle with a magnetic moment moving in an electric field $\mathcal{E}$ acquires an $\mathrm{AC}$ phase, due to the spin-orbit interaction. This AC effect has been discussed for a fluxon in type-II superconductors ${ }^{6}$ and a vortex in Josephson-junction arrays. ${ }^{7,8}$ Analogous to the spontaneous $\mathrm{AB}$ effect, an intriguing spontaneous $\mathrm{AC}$ effect due to many-body effects for electrons in onedimensional (1D) mesoscopic normal-metal rings was investigated recently. ${ }^{9,10}$ Unfortunately, in reality, an observable spontaneous AC effect is usually suppressed by the dominant $A B$ effect, because the electron also possesses charge. ${ }^{5,9}$ On the other hand, in identifying the existence of a spontaneous AC effect, statistics could play a crucial role (e.g., fermion or spinor statistics). Notice that the previously mentioned fluxons (or vortices) in type-II superconductors (or Josephson-junction arrays) can be treated as standard neutral hard-core bosons carrying magnetic moment $\boldsymbol{\mu}_{\mathrm{HB}}$. Thus, it seems natural, general, and important to consider the $\mathrm{AC}$ problem in a system of many hard-core bosons. In this paper, using a tight-binding model Hamiltonian and applying the Jordan-Wigner transformation technique, we investigate the AC effect for many hard-core bosons in 1D mesoscopic rings including the self-induced $A C$ phase. It is indicated that the spontaneous $\mathrm{AC}$ effect could exist at sufficiently low temperature in such a system with weak disorder. Moreover, the total energy and the persistent fluxon current is analytically derived for the 1D perfect lattice.

Let us consider $N_{\mathrm{HB}}$ neutral hard-core bosons in an
$N$-site $\left(N_{\mathrm{HB}}<N\right)$ 1D mesoscopic ring with radius $R$, vanishingly small width $a$ and thickness $L$, lying in the $x y$ plane. ${ }^{11}$ Each neutral hard-core boson carries a magnetic flux quantum $\boldsymbol{\Phi}_{\mathrm{HB}}$ along the $z$ direction $\left(\boldsymbol{\mu}_{\mathrm{HB}}=\boldsymbol{\Phi}_{\mathrm{HB}} L / 4 \pi\right)$. In the presence of the AC phase, $\Phi_{\mathrm{AC}}=\Phi_{\text {ext }}^{\mathrm{AC}}+\Phi_{\mathrm{I}}^{\mathrm{AC}}=\frac{\mu_{\mathrm{HB}}}{e} \oint_{C} \hat{\boldsymbol{z}} \cdot(d l \times \mathcal{E})$, where $\Phi_{\text {ext }}^{\mathrm{AC}}$ and $\Phi_{\mathrm{I}}^{\mathrm{AC}}$ are, respectively, the externally applied AC flux and self-induced $\mathrm{AC}$ flux enclosed by the ring, and $\mathcal{E}$ in the integral is the total electric field. If the local potential on sites is strong enough when compared to the boson's kinetic energy, the effective model Hamiltonian of the system can be written as ${ }^{12}$

$$
H=-\sum_{i=1}^{N}\left(t_{i+1, i} e^{i \Delta \theta_{i}} b_{i+1}^{\dagger} b_{i}+\text { H.c. }\right)+\sum_{i=1}^{N} \epsilon_{i} b_{i}^{\dagger} b_{i},
$$

where $\epsilon_{i}$ is the on-site energy, $t_{i+1, i}=t_{i, i+1}$ is the hopping matrix, $i=\sqrt{-1}, \Delta \theta_{i}$ is the phase acquired by a hard-core boson on hopping from the $i$ th site to the $(i+1)$ th site, and $\sum_{i=1}^{N} \Delta \theta_{i}=2 \pi \Phi_{\mathrm{AC}} / \Phi_{0}=2 \pi f_{\mathrm{AC}}$, with the flux quantum $\Phi_{0}=h c / e, b_{i}^{\dagger}$ and $b_{i}$ are the creation and annihilation operators of a neutral hard-core boson on the $i$ th site. These operators satisfy the following relations $^{13}$

$$
\begin{aligned}
& {\left[b_{i}, b_{j}\right]=\left[b_{i}, b_{j}^{\dagger}\right]=0 \text { for } i \neq j,} \\
& \left\{b_{i}, b_{i}^{\dagger}\right\}=1 \text { and } b_{i}^{2}=0,
\end{aligned}
$$

where [ ] and \{\} denote commutator and anticommutator, respectively. Actually, they are spinors and supposedly represent neutral particles carrying magnetic moments. Although we concentrate here on the physics particular to the AC effect, Eq. (1) is rather a general model, which represents a system of particles obeying spinor statistics that acquire an added phase when "hopping" from a site on a $1 D$ lattice to a neighboring one, and it may apply to a variety of systems.

In order to treat the above nontrivial Hamiltionian, it is crucial to introduce the Jordan-Wigner transformation, ${ }^{13}$

$$
b_{i}=f_{i} e^{i \pi \sum_{1 \leq l<i} f_{l}^{\dagger} f_{l}}, b_{i}^{\dagger}=e^{-i \pi \sum_{1 \leq l<i} f_{l}^{\dagger} f_{l}} f_{i}^{\dagger}
$$


where $f_{i}$ and $f_{i}^{\dagger}$ are the usual fermion operators that satisfy the anticommutating relations,

$$
\left\{f_{i}, f_{j}^{\dagger}\right\}=\delta_{i j},\left\{f_{i}, f_{j}\right\}=\left\{f_{i}^{\dagger}, f_{j}^{\dagger}\right\}=0,
$$

for $i=1,2, \ldots, N$. Note that if we choose the boundary condition $b_{N+1}=-b_{1}$, we could have $f_{N+1}=f_{1} e^{-i \pi N_{\mathrm{HB}}}$, with $N_{\text {нв }}$ as the total number of hard-core bosons. Consequently, Eq. (1) can be rewritten as

$$
\begin{aligned}
H= & -\sum_{i=1}^{N-1}\left(t_{i+1, i} e^{i \Delta \theta_{i}} f_{i+1}^{\dagger} f_{i}+\text { H.c. }\right) \\
& -\left(t_{1, N} e^{i\left(\Delta \theta_{N}+\pi \hat{N}_{\mathrm{HB}}\right)} f_{1}^{\dagger} f_{N}+\text { H.c. }\right)+\sum_{i=1}^{N} \epsilon_{i} f_{i}^{\dagger} f_{i} .
\end{aligned}
$$

Equation (2) is simply the Hamiltonian of noninteracting fermions on a ring threaded by a flux. Obviously, $H$ can be represented as a quasitridiagonal $N \times N$ matrix in the single-site representation,

$H=\left(\begin{array}{cccc}\epsilon_{1} & -t_{12} e^{-i \Delta \theta_{1}} & \cdots & -t_{1 N} e^{i \Delta \bar{\theta}_{N}} \\ -t_{12} e^{i \Delta \theta_{1}} & \epsilon_{2} & \cdots & \\ \cdots & \cdots & \cdots & \cdots \\ -t_{1 N} e^{-i \Delta \bar{\theta}_{N}} & & \cdots & \epsilon_{N}\end{array}\right)$,

with $\Delta \bar{\theta}_{N}=\Delta \theta_{N}+\pi N_{\mathrm{HB}}$. We now introduce a unitary transformation,

$U=\left(\begin{array}{llll}e^{-i \sum_{l=1}^{N-1} \Delta \theta_{l}} & & & \\ & e^{-i \sum_{l=2}^{N-1} \Delta \theta_{l}} & & \\ & & e^{-i \Delta \theta_{N-1}} & \\ & & & 1\end{array}\right)$

which simplifies the Hamiltonian matrix in Eq. (3) to the form

$$
\tilde{H}=U^{\dagger} H U=\left(\begin{array}{cccc}
\epsilon_{1} & -t_{12} & \cdots & -\tilde{t}_{1 N}^{*} \\
-t_{12} & \epsilon_{2} & \cdots & \\
\cdots & \cdots & \cdots & \cdots \\
-\tilde{t}_{1 N} & & \cdots & \epsilon_{N}
\end{array}\right)
$$

with $\tilde{t}_{1 N}=t_{1 N} e^{-i \pi\left(2 f_{\mathrm{AC}}+N_{\mathrm{HB}}\right)}$. This transformation makes it clear that the transformed $\tilde{H}$ depends only on the total AC phase enclosed rather than all the individual phases acquired at each step, and shows the familar result that the only effect of the flux is to modify the boundary condition. Now one can easily find that the eigenvalues of $\tilde{H}$ in Eq. (5), which is a textbook tight-binding model with flux-shifted boundary condition, is periodic in $\Phi_{A C}$ with the period $\Phi_{0}$, and so are other physical quantities. In general, we need to solve the eigenvalue problem for $\tilde{H}$ given by Eq. (5) numerically, and then calculate the total energy and persistent fluxon current for the fixed number $N_{\mathrm{HB}}$. In Fig. 1 and Fig. 2 we plot the energy spectrum, total energy, and fluxon current

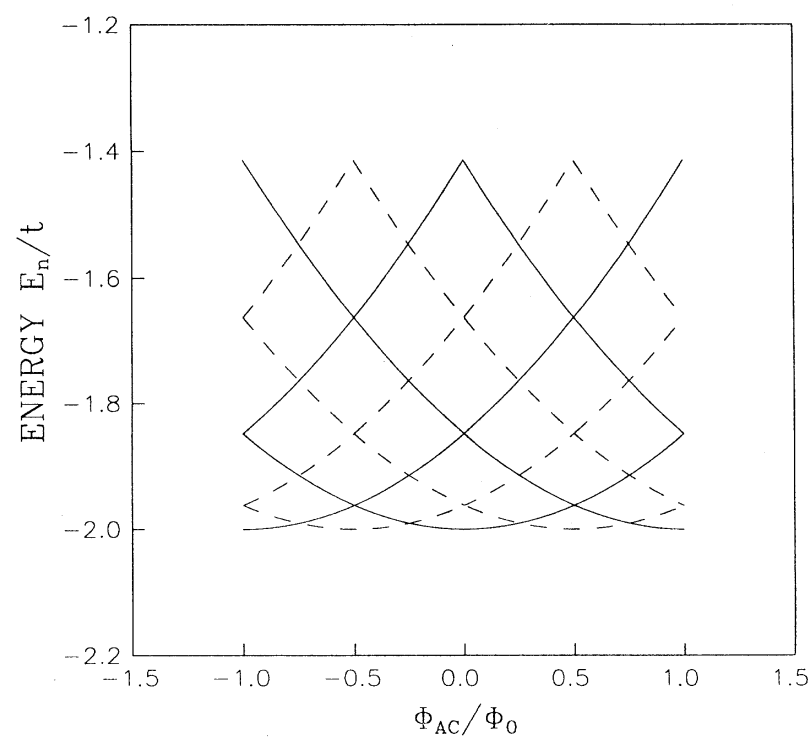

FIG. 1. The energy spectrum (first four levels) as a function of dimensionless $\mathrm{AC}$ flux $\left(\Phi_{\mathrm{AC}} / \Phi_{0}\right)$ threading the ring, calculated using the tight-binding model without disorder for $N=16, N_{\mathrm{HB}}=$ even and odd (solid and dashed curves, respectively).

as a function of the $\mathrm{AC}$ flux for a perfect lattice, i.e., $t_{i j}=t$. (Hereafter, we set $\epsilon_{i}=0$.) The energy spectrum for odd $N_{\mathrm{HB}}$ coincides with that for even $N_{\mathrm{HB}}$ if one shifts by $\Phi_{\mathrm{AC}} / \Phi_{0}=1 / 2$ along the AC flux axis. At this stage, the possibility of the spontaneous AC effect could be expected because, in the absence of the external flux, we see that the energy of the state with self-induced $\mathrm{AC}$ flux is always lower than that without $\mathrm{AC}$ flux. In fact, for the perfect lattice, the above eigenvalue problem can be solved analytically by introducing an equivalent transformation that casts the boundary factor $e^{ \pm i} \pi\left(2 f_{\mathrm{AC}}+N_{\mathrm{HB}}\right)$ in $\tilde{H}$ into the boundary condition for the wave function, i.e., $\Psi_{N+1}=\Psi_{1} e^{+i} \pi\left(2 f_{\mathrm{AC}}+N_{\mathrm{HB}}\right) .{ }^{14}$ Consequently, the energy $E_{n}$ and fluxon current $I_{n}$ of the $n$th eigenstate are derived as

$$
\begin{aligned}
E_{n} & =-2 t \cos \left[\frac{2 \pi}{N}\left(n+f_{\mathrm{AC}}\right)\right], \\
I_{n} & =\frac{\Phi_{\mathrm{HB}}}{2 \pi \hbar} \frac{\partial E_{n}}{\partial f_{\mathrm{AC}}} \\
& =\frac{2 \Phi_{\mathrm{HB}} t}{N \hbar} \sin \left[\frac{2 \pi}{N}\left(n+f_{\mathrm{AC}}\right)\right],
\end{aligned}
$$

for even $N_{\mathrm{HB}}$, and

$$
\begin{aligned}
E_{n} & =-2 t \cos \left[\frac{2 \pi}{N}\left(n+f_{\mathrm{AC}}-\frac{1}{2}\right)\right], \\
I_{n} & =\frac{2 \Phi_{\mathrm{HB}} t}{N \hbar} \sin \left[\frac{2 \pi}{N}\left(n+f_{\mathrm{AC}}-\frac{1}{2}\right)\right],
\end{aligned}
$$

for odd $N_{\mathrm{HB}}$, where $n=0, \pm 1, \pm 2, \ldots$. At zero temperature, the total energy, and fluxon current for a fixed 

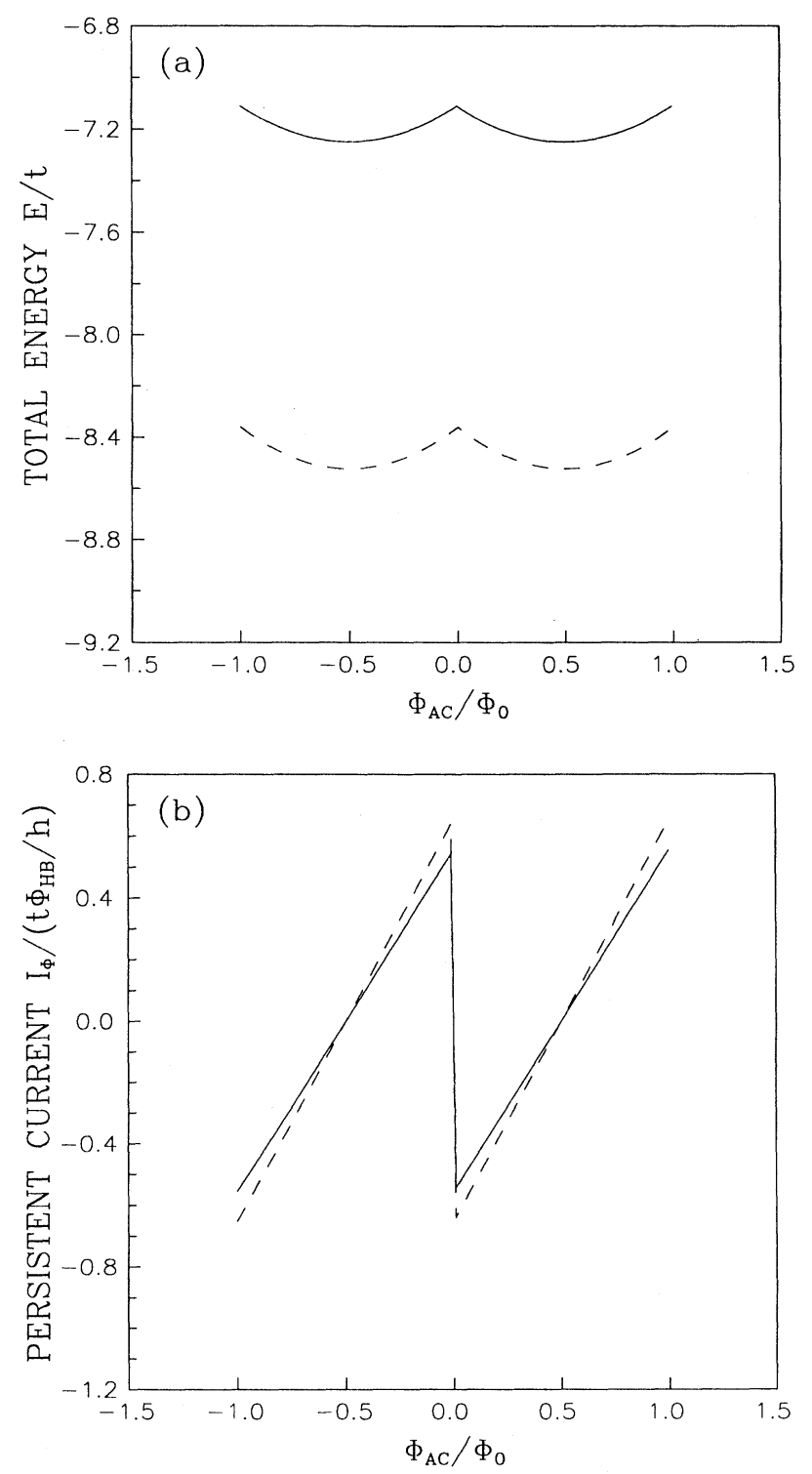

FIG. 2. The total energy $E$ (a) and the persistent current $I_{\Phi}(\mathrm{b})$ vs the AC flux within two periods for $N=16, N_{\mathrm{HB}}=4$ (solid curve) and 5 (dashed curve).

number of hard-core bosons $N_{\mathrm{HB}}$ are found to be

$$
\begin{aligned}
E & =\sum_{n} E_{n} \\
& =-2 t \frac{\sin \left(\pi N_{\mathrm{HB}} / N\right) \cos \left[(2 \pi / N)\left(f_{\mathrm{AC}}-1 / 2\right)\right]}{\sin (\pi / N)}, \\
I_{\Phi} & =\sum_{n} I_{n} \\
& =\frac{2 \Phi_{\mathrm{HB}} t \sin \left(\pi N_{\mathrm{HB}} / N\right) \sin \left[(2 \pi / N)\left(f_{\mathrm{AC}}-1 / 2\right)\right]}{\sin (\pi / N)},
\end{aligned}
$$

with $f_{\mathrm{AC}}$ being in the range $[0,1)$.
So far, we have obtained the "macroscopic" current $I_{\Phi}$ from the quantum calculation. Note that the reduced flux $f_{\mathrm{AC}}$ is also a "macroscopic" parameter and, according to conventional wisdom, it is determined from classical electrodynamical considerations, ${ }^{4,9,12}$ which, provided that the self-induced $\mathrm{AC}$ flux $f_{\mathrm{AC}}^{(I)}$ is negligible, could be independent of the detailed geometry of the ring. In fact, in the absence of the external flux and at extremely low temperature, the $f_{\mathrm{AC}}^{(I)}$ induced by $I_{\Phi}$ itself cannot be neglected. Because of this, it is reasonable and acceptable to consider the current-induced flux $f_{\mathrm{AC}}^{(I)}$ and calculate it based upon classical considerations and some average notion.

It is well known that an electric field $\varepsilon_{n}$ is induced when a particle carrying a magnetic flux moves with velocity $\boldsymbol{v}_{n}$. According to Faraday's law, $\nabla \times \boldsymbol{\varepsilon}_{n}=-\frac{1}{c} \frac{\partial \boldsymbol{b}}{\partial t}$, we are able to obtain $\varepsilon_{n}=\frac{1}{c} b \times v_{n}=\frac{2 \pi R}{\Phi_{\mathrm{HB}} c} b \times I_{n}$ with $\boldsymbol{b}=\boldsymbol{\Phi}_{\mathrm{HB}} /(2 \pi R a)$ as the effective fluxon density of an individual particle. ${ }^{15}$ The total electric field generated by the fluxon current inside the ring can be found,

$$
\mathcal{E}=\sum_{n} \varepsilon_{n}=-\frac{1}{a c} I_{\Phi} \hat{\boldsymbol{r}}
$$

where $\hat{\boldsymbol{r}}$ is a unit radial vector. The induced AC flux is then found to be

$$
f_{\mathrm{AC}}^{(I)}=\frac{\mu_{\mathrm{HB}} \mathcal{E}_{r} R}{\hbar c}=-\frac{\Phi_{\mathrm{HB}} L R}{4 \pi a \hbar c^{2}} I_{\Phi} .
$$

In the absence of the external AC flux, Eqs. (9) and (10) lead to a self-consistent solution $f_{\mathrm{AC}}^{(s)}$ for the self-induced $\mathrm{AC}$ flux. For very small $a$, to order of $(a / R)^{2}, f_{\mathrm{AC}}^{(s)}$ can be approximated as

$$
f_{\mathrm{AC}}^{(s)} \approx \frac{1}{2}\left[1+\frac{a N^{2} \hbar^{2} c^{2} \sin (\pi / N)}{\Phi_{\mathrm{HB}}^{2} t L R \sin \left(\pi N_{\mathrm{HB}} / N\right)}\right]^{-1}
$$

On the other hand, the total energy of the whole system consists of two parts: the energy of the particles in the ring $E$, and the energy of the electric field $E_{\mathcal{E}}$, i.e., $E_{\mathbf{T}}=$ $E+E_{\mathcal{E}}$, where

$$
E_{\mathcal{E}}=\frac{1}{8 \pi} \int \mathcal{E}^{2} d^{3} x=\frac{4 \pi^{2} a \hbar^{2} c^{2}}{\Phi_{\mathrm{HB}}^{2} L R} f_{\mathrm{AC}}^{2} .
$$

It is straightforward to find that the total energy $E_{\mathrm{T}}$ reaches its minimum just at $f_{\mathrm{AC}}=f_{\mathrm{AC}}^{(s)}$, i.e., $\left.\frac{\partial E_{\mathrm{T}}}{\partial f_{\mathrm{AC}}}\right|_{s}=$ $0,\left.\frac{\partial^{2} E_{T}}{\partial f_{\mathrm{AC}}^{2}}\right|_{s}>0$, which implies that the AC flux state with the spontaneous fluxon current is in fact the stable ground state of the system. We have, therefore, the remarkable result that in the absence of an external electric field the AC flux, $\Phi_{\mathrm{AC}}=f_{\mathrm{AC}}^{(s)} \Phi_{0}$, and the corresponding fluxon current can be self-sustained in the system regardless of whether the number of hard-core bosons $N_{\mathrm{HB}}$ is even or odd. This quite different from the case of a fully polarized normal-metal ring, where the flux state carrying a spontaneous spin current is only possible for an even number of electrons. ${ }^{9,10}$ Note that, if the width $a$ of the ring approaches zero, the self-sustained AC flux 
approaches a finite constant $\frac{1}{2}$, whereas the flux current $I_{\Phi}^{(s)}$ approaches zero.

We wish to point out that, in analogy to electron systems, ${ }^{16}$ all hard-core bosons in one-dimensional disordered systems are exponentially localized. We will speak of weakly or strongly disordered rings depending on whether the dimensionless localization length $\tilde{l}$ is larger or smaller than the dimensionless circumference $N$ of the ring. For the above circular geometry, we are able to define the crossover from weak to strong disorder by $N \approx \tilde{l}$. If we choose random on-site energy $\epsilon_{i}$ uniformly distributed between $-W / 2$ and $+W / 2$, and set the hopping matrix element $t_{i j}=t$, the localization length $\tilde{l}$ will be uniquely determined by the disorder parameter $W / t$. For large $W$, the hard-core bosons are tightly bound with energies close to their on-site energies $\epsilon_{i}$. Numerical calculations demonstrate that when $W>2 \pi t$, the localization length is less than five lattice constants. It is thus expected that the spontaneous AC effect will be destroyed by strong disorder, but will survive with weak disorder in mesoscopic systems. As for the effects of a finite temperature, one can similarly define a characteristic temperature $T^{*}=\left(\frac{2 t}{k_{B} \pi N}\right) \sin \left(N_{\mathrm{HB}} \pi / N\right) .{ }^{16}$ The persistent current decreases dramatically with $T / T^{*}$ at $T>T^{*}$, however, it is insensitive to temperature at $T<T^{*}$.

Finally, it may be useful to discuss briefly the possibility for experimental observation of the expected spontaneous AC effect. Consider an annular type-II superconducting sample in the dilute mixed state with $a \sim \lambda \sim \xi$ and the external magnetic field applied along the $z$ axis, where $\lambda$ and $\xi$ are, respectively, the penetration length and the superconducting coherence length. In the ideal case, i.e., $f_{\mathrm{AC}}^{(s)} \sim 1 / 2$, and at very low temperature, the possible maximum signal of the radial voltage, due to the spontaneous $\mathrm{AC}$ effect, may be roughly estimated as $V_{\mathrm{AC}}^{(s)} \sim \mathcal{E}^{(s)} \lambda \sim \frac{\hbar c}{2 \mu_{\mathrm{HB} R}} \lambda \sim \frac{e}{R}\left(\frac{\lambda}{L}\right) \sim 10^{-6}(\mathrm{~V})$ if $R \sim 10 \mu \mathrm{m}$ and $L \sim 10 \lambda$ are chosen, which could be observed by some suitably designed experiments.

We gratefully acknowledge helpful discussions with Professor Jinming Dong, Professor Zhao-Bin Su, Dr. Qinwei Shi and Dr. J. Wang. In particular, great thanks are extended to Dr. P. K. Mackeown for his critical reading of our manuscript. This work was supported by RGC grant of Hong Kong and CRCG research grant at the University of Hong Kong.
${ }^{1}$ Y. Aharonov and D. Bohm, Phys. Rev. 115, 485 (1959).

${ }^{2}$ M. Büttiker, Y. Imry, and R. Landauer, Phys. Lett. 96A, 365 (1983); R. Landauer and M. Büttiker, Phys. Rev. Lett. 54, 2049 (1985).

${ }^{3}$ L. P. Lévy, G. Dolan, J. Dunsmuir, and H. Bouchiat, Phys. Rev. Lett. 64, 2074 (1990); V. Chandrasekhar, R. A. Webb, M. J. Brady, M. B. Ketchen, W. J. Gallagher, and A. Kleinsasser, ibid. 67, 3578 (1991); D. Mially, C. Chapelier, and A. Benoit, ibid. 70, 2020 (1993).

${ }^{4}$ D. Wohlleben, M. Esser, P. Freche, E. Zipper, and M. Szopa, Phys. Rev. Lett. 66, 3191 (1991); Mod. Phys. Lett. B 6, 1481 (1992).

${ }^{5}$ Y. Aharonov and A. Casher, Phys. Rev. Lett. 53, 319 (1984); C. R. Hagen, ibid. 64, 2347 (1990).

${ }^{6}$ R. Reznik and Y. Aharonov, Phys. Rev. D 40, 4178 (1989).

${ }^{7}$ B. J. van Wees, Phys. Rev. Lett. 65, 255 (1990); T. P. Orlando and K. A. Delin, Phys. Rev. B 43, 8717 (1991).

${ }^{8}$ W. J. Elion, J. J. Wachters, L. L. Sohn, and J. E. Mooij, Phys. Rev. Lett. 71, 2311 (1993).

${ }^{9}$ M. Y. Choi, Phys. Rev. Lett. 71, 2987 (1993).

${ }^{10}$ Jian-Xin Zhu and Z. D. Wang, J. Phys. Condens. Matter
6, L329 (1994)

${ }^{11}$ For the fluxon systems, the site may be roughly viewed as the pinning site as long as $N_{\mathrm{HB}}<N ; a$ and $L$ are less than the corresponding dimension of a fluxon.

${ }^{12}$ Isaac Goldhirsch, Dror Lubin, and Yuval Gefen, Phys. Rev. Lett. 67, 3582 (1991).

${ }^{13}$ E. Fradkin, Field Theories of Condensed Matter Systems (Addison-Wesley, Redwood City, 1991).

14 There is an alternative way leading to the analytical solution. By introducing the specific unitary transformation $\bar{U}_{j j^{\prime}}=e^{i(N-j) \Delta \tilde{\theta}} \delta_{j j^{\prime}}$, we are able to arrive at an equivalent Hamiltonian in the form of Eq. (1) with $b_{i}$ being replaced by $f_{i}$ and $\Delta \theta_{i}=\Delta \tilde{\theta}=\left(\frac{2 \pi}{N}\right) f_{\mathrm{AC}}$ for even $N_{\mathrm{HB}}$ or $\left(\frac{2 \pi}{N}\right)\left(f_{\mathrm{AC}}-\frac{1}{2}\right)$ for odd $N_{\mathrm{HB}}$, which can be easily diagonalized.

${ }^{15} \mathrm{M}$. Tinkham, Introduction to Superconductivity (Pergamon, Oxford, 1969); Z. D. Wang and C. S. Ting, Phys. Rev. B 46, 284 (1992).

${ }^{16}$ Ho-Fai Cheung, Yuval Gefen, Eberhard K. Riedel, and WeiHeng Shih, Phys. Rev. B 37, 6050 (1988). 